University of Wollongong

Research Online

Faculty of Engineering - Papers (Archive)

Faculty of Engineering and Information

Sciences

$1-1-2011$

\title{
Role of vanguard counter-potential in terahertz emission due to surface currents explicated by three-dimensional ensemble Monte Carlo simulation
}

David Cortie

University of Wollongong, dlc422@uowmail.edu.au

Roger A. Lewis

University of Wollongong, roger@uow.edu.au

Follow this and additional works at: https://ro.uow.edu.au/engpapers

Part of the Engineering Commons

https://ro.uow.edu.au/engpapers/2927

\section{Recommended Citation}

Cortie, David and Lewis, Roger A.: Role of vanguard counter-potential in terahertz emission due to surface currents explicated by three-dimensional ensemble Monte Carlo simulation 2011, 155328-1-155328-9. https://ro.uow.edu.au/engpapers/2927 


\title{
Role of vanguard counter-potential in terahertz emission due to surface currents explicated by three-dimensional ensemble Monte Carlo simulation
}

\author{
D. L. Cortie* and R. A. Lewis ${ }^{\dagger}$ \\ Institute for Superconducting and Electronic Materials, University of Wollongong, Wollongong, New South Wales 2522, Australia
}

(Received 18 July 2011; revised manuscript received 28 September 2011; published 31 October 2011)

\begin{abstract}
The discovery that short pulses of near-infrared radiation striking a semiconductor may lead to emission of radiation at terahertz frequencies paved the way for terahertz time-domain spectroscopy. Previous modeling has allowed the physical mechanisms to be understood in general terms but it has not fully explored the role of key physical parameters of the emitter material nor has it fully revealed the competing nature of the surface-field and photo-Dember effects. In this context, our purpose has been to more fully explicate the mechanisms of terahertz emission from transient currents at semiconductor surfaces and to determine the criteria for efficient emission. To achieve this purpose we employ an ensemble Monte Carlo simulation in three dimensions. To ground the calculations, we focus on a specific emitter, InAs. We separately vary distinct physical parameters to determine their specific contribution. We find that scattering as a whole has relatively little impact on the terahertz emission. The emission is found to be remarkably resistant to alterations of the dark surface potential. Decreasing the band gap leads to a strong increase in terahertz emission, as does decreasing the electron mass. Increasing the absorption dramatically influences the peak-peak intensity and peak shape. We conclude that increasing absorption is the most direct path to improve surface-current semiconductor terahertz emitters. We find for longer pump pulses that the emission is limited by a newly identified vanguard counter-potential mechanism: Electrons at the leading edge of longer laser pulses repel subsequent electrons. This discovery is the main result of our work.
\end{abstract}

DOI: $10.1103 /$ PhysRevB.84.155328

PACS number(s): 42.70.Nq, 42.72.Ai, 73.20.Mf, 78.20.Bh

\section{INTRODUCTION}

Methods for the generation and detection of electromagnetic radiation in the terahertz frequency regime are of great current interest. ${ }^{1-10}$ A key development has been the production of terahertz pulses on the sub-picosecond time scale by excitation of a suitable target using sub-picosecond pulses from a near-infrared laser., ${ }^{3,11}$ The availability of short pulses of terahertz radiation has led to the development of terahertz time-domain spectroscopy. ${ }^{7,12}$ In contrast to many other spectroscopic techniques, which are sensitive only to the intensity of the radiation under investigation, time-domain spectroscopy directly detects the amplitude and phase of the electromagnetic wave. This enables the direct determination of the real and imaginary components of the optical constants. $^{7}$

There are three main ways by which pulsed terahertz radiation is produced under sub-picosecond optical excitation. ${ }^{13}$ First, if the pump radiation energy exceeds the band gap, it may produce electron-hole pairs, which in turn are accelerated under an applied potential. This is the phenomenon of photoconductivity. ${ }^{14,15}$ This was the first method developed, has been studied extensively, and is deployed widely, but is limited by catastrophic electrical breakdown and thermal runaway. ${ }^{16,17}$ Second, if an electro-optic crystal is used as the emitter, the non-linear mixing of the different frequency components of the pump radiation may produce differencefrequency mixing at terahertz frequencies. ${ }^{18-20}$ This method is simple but relatively inefficient. ${ }^{18}$ Third, photocarriers produced by above-band-gap pump radiation may give rise to transient currents that in turn give rise to terahertz radiation without the need for an externally applied bias. ${ }^{11,21,22}$ This results in a relatively efficient, robust terahertz emitter. ${ }^{13,21,22}$ Such transient currents are the subject of the present paper.
The transient currents at the surfaces of semiconductors may be divided into two classes. These may be classified as drift and diffusion currents. ${ }^{13,23}$ If there is an inbuilt electric field at the surface of the semiconductor, this will cause electrons and holes to drift in opposite directions perpendicular to the surface, ${ }^{11}$ producing an instantaneous dipole. ${ }^{23}$ Such currents are also referred to as surface or surface-field currents. If there is a difference in the mobility of electrons and holes, the two types of charge carriers will diffuse away from the surface at different rates, again producing an instantaneous dipole. ${ }^{23,24}$ The differential diffusion of electrons and holes is known as the Dember effect; if the charge carriers involved have been produced by photoexcitation the phenomenon is known as the photo-Dember effect. ${ }^{25}$ It tends to be that the diffusion, or photo-Dember, effect is more prominent in narrow-gap semiconductors, such as InAs, whereas the drift, or surface-field effect, is more prominent in wider-gap semiconductors, such as GaAs. ${ }^{13,23,26}$ It should be emphasized that this identification of two distinct processes is a simplification. In many cases, both drift and diffusion occur together, even competing, ${ }^{26}$ and a full description needs to accommodate both effects on the carrier dynamics leading to terahertz emission. In our approach, to be described in detail in Sec. II, we do not specifically or separately consider these the two processes, but instead carry out a full carrier dynamics simulation in three dimensions based on first principles. In contrast to some previous treatments, the photo-Dember voltage is not contrived as an external boundary condition based on the steady-state solution, ${ }^{27}$ but naturally arises as a self-consistent feature of the dynamic diffusion in our three-dimensional simulation.

In this paper we model the motion of photocreated charge carriers from fundamental principles. One approach would be to employ the Boltzmann transport equation. ${ }^{28}$ However, since the excitation occurs on a similar time scale to the scattering 
processes, a hydrodynamic treatment is questionable. ${ }^{23,29} \mathrm{~A}$ better approach is to carry out a Monte Carlo transport simulation in three dimensions treating scattering processes explicitly from quantum mechanics. Such models have already enjoyed a great deal of success, having been able to demonstrate, in agreement with experimental studies: (a) The higher intensity of terahertz emission from InAs than from GaAs; ${ }^{23}$ (b) the reversal of the terahertz field with reversal of doping ( $p$ - to $n$-) type in GaAs but not in InAs, due to a preponderance of the photocarrier drift in the former but diffusion in the latter; ${ }^{23}$ (c) the effect of a magnetic field in rotating the radiating dipole to a more favorable orientation for terahertz emission; ${ }^{30}$ and (d) observed shapes of the emitted wave form under different external fields and excitation densities. ${ }^{25,31}$

We carry out a full Monte Carlo carrier dynamics simulation for many different parameter configurations to identify the influence of each parameter on the generated terahertz field. To ground the calculations, we use as a starting point the material InAs, for which many experimental data exist. We then vary the parameters one at a time to explicate their particular contributions. In some cases, such as dielectric constant, the parameter is not readily amenable to change in practice, so the simulation is more in the nature of a gedanken experiment. In this way we identify which parameters are important and which are less significant in controlling the terahertz emission.

The paper is arranged as follows. In Sec. II an overview of our approach to the Monte Carlo method for transport simulation is given. In Sec. III the effects of varying various parameters are shown and interpreted. The newly identified vanguard counter-potential effect is discussed in Sec. IV. In Sec. V, we draw our conclusions. The Appendix provides more specific details regarding the numerical calculations in the model.

\section{METHOD}

We adopt a classical Monte Carlo approach ${ }^{32}$ that follows three basic steps: ${ }^{33,34}$ (1) A particle is introduced into the simulation area; (2) during a small time interval $d t$, the particle moves ballistically; (3) at the end of the time interval $d t$, a scattering mechanism is selected; (4) the particle's momentum is changed accordingly. The steps (2)-(4) are then repeated for as many time intervals as required. Rather than consider only the behavior of a single particle, we consider many interacting particles simultaneously, the so-called ensemble Monte Carlo method. ${ }^{34}$ This is the same general approach adopted in previous work applying Monte Carlo methods to terahertz generation ${ }^{23,25,31}$ and semiconductor device simulation. ${ }^{34}$ Some clear principles have been demonstrated in work by previous groups ${ }^{23,25,27}$ in this field and we adopt these here. First, the charge-carrier diffusion and the electric field distribution must be solved self-consistently so that the electric field is updated to reflect the new charge distribution after each time interval; ${ }^{23,29}$ it is insufficient to consider only the static or external field. Second, the energy and the momentum relaxation of the charge carriers must incorporate appropriate scattering mechanisms; ${ }^{23,25,29}$ polar optical phonon scattering is considered to be most important scattering mechanism ${ }^{35}$ (these scattering probabilities are derived from Fermi's golden rule $^{34}$ and have been simplified here in Table II for the case of parabolic bands). Third, charge carrier motion between scattering events may be considered semiclassically. ${ }^{23,27,29,31}$ By this is meant that classical (Newtonian) mechanics may be used, provided the effective mass given by the band structure is employed. Fourth, the emission of terahertz radiation in the far field is proportional to the time derivative of the current density. ${ }^{23,29-31,36}$ Details of the model are given in the Appendix.

\section{RESULTS AND DISCUSSION}

\section{A. Standard semi-insulating case: Consistency with previous modeling}

To facilitate comparison between the previous work and the results presented here, we define a standard case of $n$-type seminsulating InAs (with the parameters given in Table I), which is excited by a laser pulse consisting of photons with an energy of $1.55 \mathrm{eV}$ distributed in time by a Gaussian pulse with a full width at half maximum (FWHM) of $100 \mathrm{fs}$, consistent with the parameters employed in simulations in Ref. 23 .

We set the surface potential to lie very near the conduction band edge of InAs, to give a band pinning of $0.1 \mathrm{~V}$. We choose the positive sign to reflect a $p$-type surface which forms an $n$-type accumulation layer in the first few atomic layers. We use time steps of $10 \mathrm{fs}$ and allow the simulation to run for an initial $10 \mathrm{ps}$ to allow (dynamic) equilibrium to be obtained before the laser strike. Analysis of the $\langle\mathbf{J}(t)\rangle$ data shows that at an elapsed time of 10 ps the system is essentially in equilibrium, and the surface potential has yielded a small accumulation layer near the surface, as expected. We then allow the laser to strike. This initiates a highly damped plasma oscillation that gives rise to terahertz emission. In presenting the data from the simulation, we give values of $d\langle\mathbf{J}(t)\rangle / d t$ averaged over all carriers and positions, and call this the terahertz electric field. For ease of comparison in further simulations when we vary materials parameters, we set the peak-peak amplitude of this emission under standard conditions to 1.0. The subsequent results are presented normalized to this unit.

TABLE I. Physical parameters of InAs used in the model. These are the same as Ref. 23, with the addition of the speed of sound.

\begin{tabular}{lc}
\hline \hline Parameter & Value \\
\hline Doping density, $n=n_{i}=p_{i}\left(\mathrm{~m}^{-3}\right)$ & $2 \times 10^{21}$ \\
Band gap, $E_{g}(\mathrm{eV})$ & 0.35 \\
Low-frequency dielectric constant, $\epsilon(0) / \epsilon_{0}$ & 15.5 \\
High-frequency dielectric constant, $\epsilon(\infty) / \epsilon_{0}$ & 12.25 \\
Effective electron mass in $\Gamma$ valley, $m_{e} / m_{0}$ & 0.022 \\
Effective hole mass, $m_{h} / m_{0}$ & 0.41 \\
Effective electron mass in $L$ valley, $m_{e} / m_{0}$ & 0.29 \\
$L-\Gamma$ valley energy offset, $E_{L-\Gamma}(\mathrm{eV})$ & 0.73 \\
Mass density, $\left.\rho(\mathrm{kg} \mathrm{m})^{-3}\right)$ & 5667 \\
Photon absorption coefficient, $\alpha\left(\mathrm{m}^{-1}\right)$ & $6.5 \times 10^{6}$ \\
LO phonon energy, $\hbar \omega_{\mathrm{LO}}(\mathrm{eV})$ & 0.03 \\
Speed of sound, $v_{s}(\mathrm{~m} / \mathrm{s})$ & 3830 \\
Deformation potential, $\mathrm{D}_{L \Gamma}=D_{t} K(\mathrm{eV} / \mathrm{m})$ & $1.4 \times 10^{10}$ \\
Laser photon energy, $E_{\mathrm{photon}}(\mathrm{eV})$ & 1.55 \\
\hline \hline
\end{tabular}




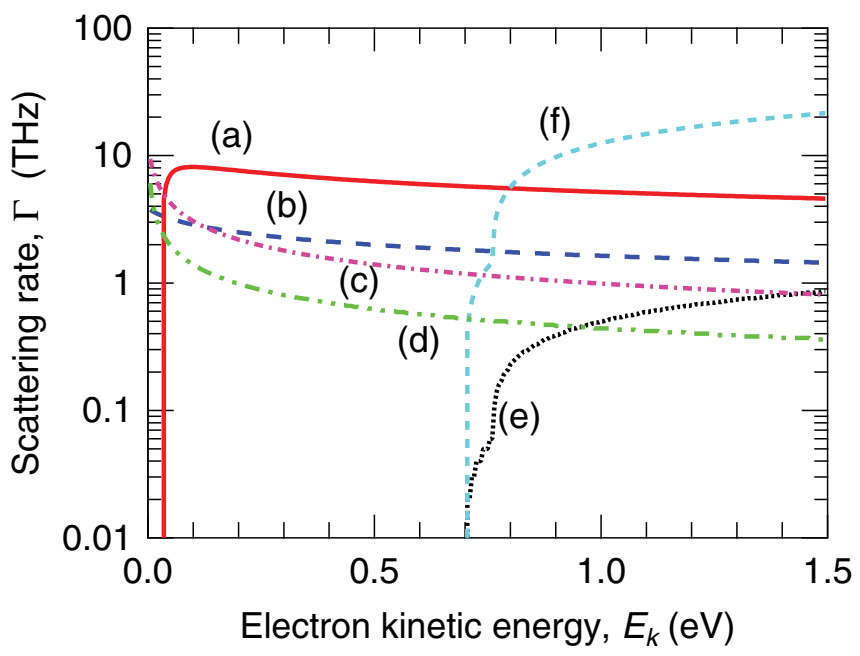

FIG. 1. (Color online) The contribution of several distinct mechanisms to electron scattering in InAs, presented as a function of electron energy. (a) Polar optical phonon emission. (b) Polar optical phonon absorption. (c) Upper limit for carrier-carrier scattering. (d) Ionized-impurity scattering. (e) Intervalley scattering using a deformation potential of $1.4 \times 10^{10} \mathrm{eV} / \mathrm{m}$ (Ref. 23). This is the value of deformation potential adopted here (see Table I). (f) Intervalley scattering, calculated using a relatively large deformation potential of $10 \times 10^{10} \mathrm{eV} / \mathrm{m}$ (Refs. 25 and 35).

\section{B. Scattering}

Figure 1 summarizes the effective scattering rates of various mechanisms used in the simulations for electrons in the central valley. The effect of the scattering mechanisms on the emission of terahertz radiation is given in Fig. 2. We see in Fig. 2 that the scattering mechanisms have very little effect on the terahertz emission from InAs. This is particularly so at very brief times. So the primary peak, at about $0.15 \mathrm{ps}$, and the secondary dip, at about $0.3 \mathrm{ps}$, are only very slightly affected. The next peak, at about $0.5 \mathrm{ps}$, is affected more strongly.

We now make more specific comments about particular scattering mechanisms. Overall, polar optical phonon scattering has the largest effect. Carrier-carrier scattering has a negligible effect for the high-energy electron-hole pairs produced by photoexcitation, but on the other hand may be the dominant scattering mechanism for the low-energy cold carriers. Ionized-impurity scattering is relatively unimportant at the doping levels of interest. The importance of intervalley scattering depends crucially on the value of the optical deformation potential parameter. Intervalley scattering has a small effect for the range of values for deformation potential generally accepted in the literature, $(1-3) \times 10^{10} \mathrm{eV} / \mathrm{m} \cdot{ }^{37,38}$ We have employed the value of $1.4 \times 10^{10} \mathrm{eV} / \mathrm{m}$ (Table I). Values as high as $10 \times 10^{10} \mathrm{eV} / \mathrm{m}$ have been used on occasion. ${ }^{25,35}$ Such high values of deformation potential have a dramatic effect on the overall scattering rate, causing carriers to be scattered almost instantaneously (within $100 \mathrm{fs}$ ) to satellite valleys. ${ }^{25}$ This is not the case in our simulations, which show a low probability for scattering to satellite valleys on these time scales.

The key result is that the pure electrodynamic simulation, with no scattering at all, replicates the important features of

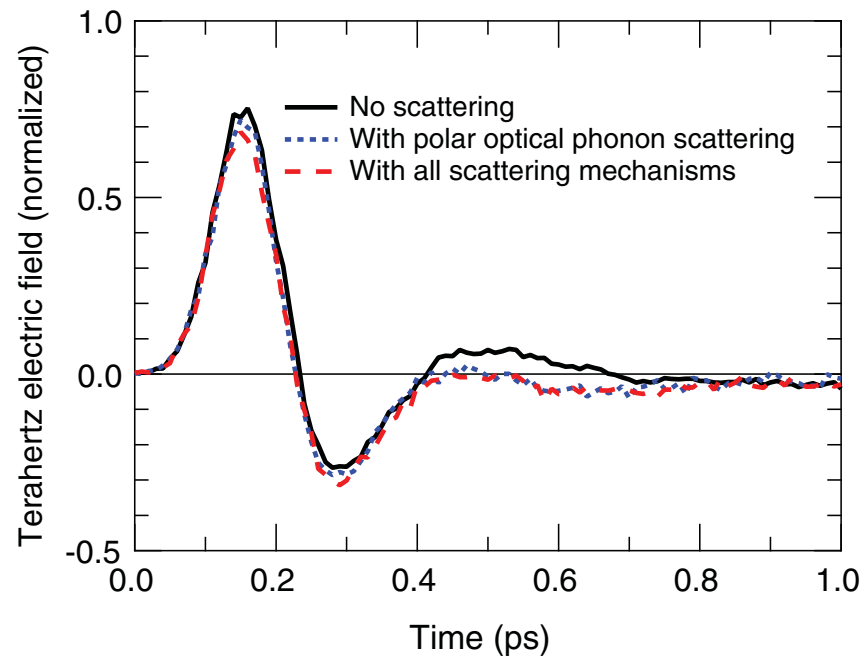

FIG. 2. (Color online) The minor role played by scattering mechanisms in the emission of terahertz radiation from InAs. The purely electrodynamic simulation (full curve), with no scattering at all, replicates the main features of the full Monte Carlo simulation with all scattering mechanisms included (dashed curve). The main scattering mechanism is polar optical phonon scattering (dotted curve). Very little additional change occurs on introducing the three additional mechanisms of carrier-carrier, intervalley, and ionizedimpurity scattering.

the simulation incorporating all the scattering mechanisms. The features reproduced include both the peak shape and the peak intensity. The explanation for this lies primarily in the low scattering rates in InAs (Fig. 1) and low scattering angle distribution for LO events (Table II). A carrier, on average, is not scattered more than 20 times in a picosecond, as may be seen from Fig. 1, and most of these are LO phonon events. Concurrently LO scattering has an angular distribution peaked strongly toward low angles so that, on average, absorption and emission events do not have a dramatic effect on electron momentum. ${ }^{39}$ Hence the transport can be considered to be almost collisionless on these time scales. (We note that this does not hold for GaAs, as our detailed simulations, to be presented elsewhere, demonstrate.)

\section{Dielectric constant}

The dielectric constant $\epsilon$ is not a parameter that can easily be varied in practice. So the nature of the discussion here is in the spirit of a gedanken experiment. It may be seen in Fig. 3 that varying the high-frequency dielectric constant $\epsilon(\infty)$ over a rather large range, from 6 to 14, changes the emitted terahertz field by less that $10 \%$. However, a noticeable change in the time signature, and consequently the bandwidth of the emitted terahertz radiation, may be observed.

\section{Band gap}

The discussion here applies to narrow-gap semiconductors generally. As the band gap is decreased, the emitted terahertz field increases. An increase of about $40 \%$ is seen in decreasing the band gap from $1.0 \mathrm{eV}$ to $0.3 \mathrm{eV}$ (Fig. 4). The effect can be understood in terms of the separation of the bands in our simple parabolic model and the effect that this has on the excess energy 
TABLE II. Scattering mechanisms employed in the model and the formulation used for each (Refs. 33 and 34). The azimuthal angle ( $\phi$ ) distribution is isotropic for each mechanism.

\begin{tabular}{|c|c|c|c|}
\hline $\begin{array}{l}\text { Scattering } \\
\text { Mechanism }\end{array}$ & $\begin{array}{c}\text { Scattering } \\
\text { Probability }(1 / \mathrm{s})\end{array}$ & $\begin{array}{c}\theta \\
\text { Spread }\end{array}$ & $\begin{array}{l}\text { Final } \\
\text { Energy }\end{array}$ \\
\hline Polar optical phonon & $\frac{e^{2} \sqrt{m^{*}} \omega_{p}}{\sqrt{2} \hbar}\left(\frac{1}{\epsilon(\infty)}+\frac{1}{\epsilon(0)}\right) \ln \left(\frac{E^{1 / 2}+E^{\prime 1 / 2}}{E^{1 / 2}-E^{\prime 1 / 2}}\right) \frac{f_{B E}\left(\hbar \omega_{p}\right)}{E^{1 / 2}}$ & $P(\theta)=\frac{E E^{\prime} \sin (\theta) d \theta}{E+E^{\prime}-2 \sqrt{E E^{\prime}} \cos (\theta)}$ & $E_{i} \pm \hbar \omega_{p}$ \\
\hline Intervalley & $\frac{\left(D_{t} K\right)^{2} m^{* 3 / 2} Z_{f}}{\sqrt{2} \pi \rho \hbar^{3} \omega_{i}}\left(E \pm \hbar \omega_{i}-\Delta E_{f i}\right)^{1 / 2} f_{B E}\left(\hbar \omega_{p}\right)$ & Isotropic & $E_{i} \pm E(L-\Gamma) \pm \hbar \omega_{p}$ \\
\hline Ionized-impurity & $\frac{2^{5 / 2} \pi n_{i} Z^{2} e^{4}}{\epsilon(0)^{2} \epsilon_{\beta}^{2} m^{1 / 2}} \frac{E^{1 / 2}}{\left(1+4 E / \epsilon_{\beta}\right)}$ & Isotropic & $E_{i}$ \\
\hline Carrier-carrier & $\frac{2^{5 / 2} \pi n_{M} Z^{2} e^{4}}{\epsilon(0)^{2} \epsilon_{\beta}^{2} m^{1 / 2}} \frac{E^{1 / 2}}{(1+4 E / \epsilon \beta)}$ & Isotropic & $E_{i}$ \\
\hline
\end{tabular}

partitioned between the electron and holes; see Eq. (A6). So a decrease in band gap will mean more energy from the excitation photon is available to accelerate the photocreated dipole pair. Of course, the increase in emission on decrease of band gap can only continue until the band gap is reduced to zero. This may be possible in some zero-gap materials, such as spin-gapless semiconductors, ${ }^{40} \mathrm{GaAs}_{1-y} \mathrm{Bi}_{y}$ with high enough Bi doping, ${ }^{41}$ or graphene. Looked at the other way, the same effect could be realized by increasing the photon frequency of the excitation pulse. It is interesting to note that a threefold increase in excess energy does not lead to a threefold increase in terahertz emission: The overall energy conversion efficiency drops for lower band gaps due to the combination of increased scattering and the vanguard counter-potential effect discussed in Sec. IV. This is relevant in understanding why InSb, which has a narrower gap than InAs, is reported to be a less efficient emitter at room temperature. ${ }^{24,39}$

\section{E. Surface potential}

The dark surface potential arises from equilibrium, immobile charge trapped in discrete, surface-localized states. All previous simulations have implicitly treated this as a constant on picosecond time scales. This is reasonable, based on the experimental evidence that the surface charging of states after

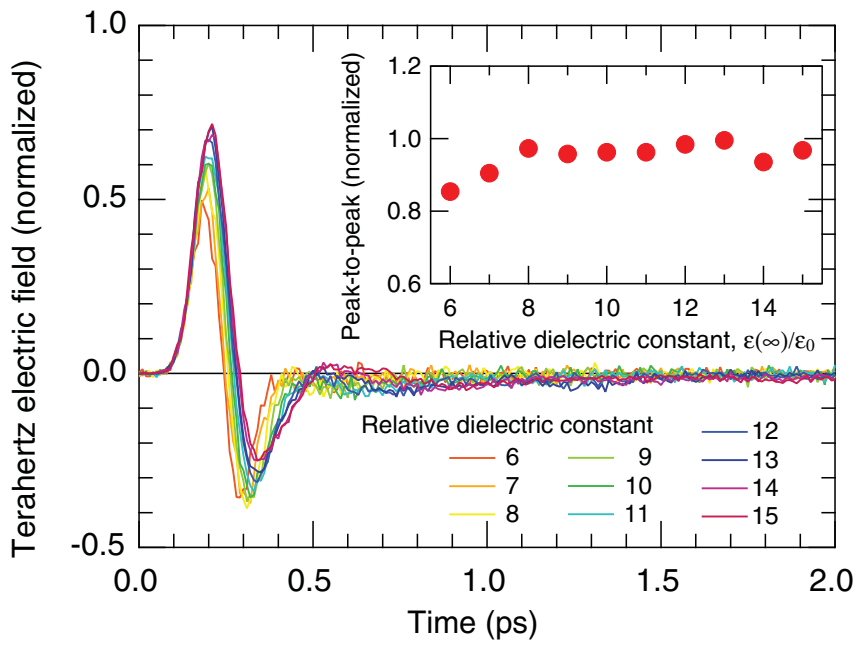

FIG. 3. (Color online) Role of the dielectric constant in the emission of terahertz radiation from InAs. Changing the dielectric constant has little effect on the amount of terahertz radiation emitted; however, the bandwidth is affected rather strongly. cleaving a crystal is a gradual process proceeding on the time scale of hours. ${ }^{42}$

As in Ref. 23, we find that the surface potential causes an equilibrium accumulation layer to form in the system before the arrival of laser pulse and this accumulation layer is sensitive to the value of the surface potential. However, the photocreated carriers are largely insensitive to this potential, due their high kinetic energy. Many escape quickly into the depth of the material, where the surface electric field is screened by existing cold carriers. These characteristics explain why InAs is primarily a photo-Dember emitter and show how robust the photo-Dember effect is.

The linear dependence of terahertz emission on surface potential we observe (Fig. 5) is consistent with the simplified model presented by Zhang. ${ }^{11}$ That model implies an almost linear dependence of terahertz emission on surface potential.

\section{F. Effective mass}

In our simple parabolic model of the band structure, the effective mass parameter describes the curvature of the $\Gamma, L$, and $X$ valleys. Decreasing the effective mass in the $\Gamma$ valley leads to a dramatic increase in terahertz emission (Fig. 6), since it strongly increases the electron mobility. In practice, it is not

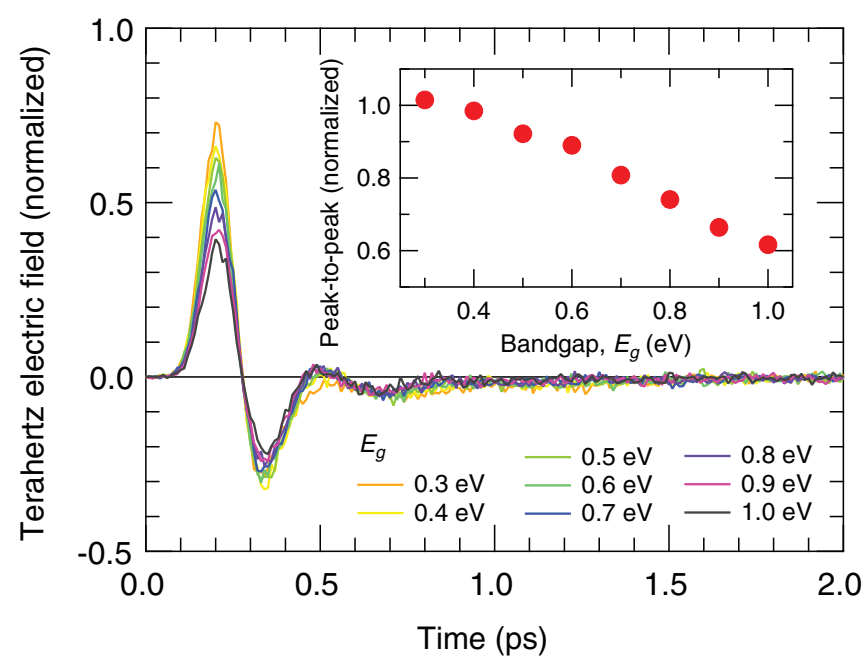

FIG. 4. (Color online) Role of band gap in the emission of terahertz radiation from InAs. Reducing the band gap strongly increases the terahertz emission. 


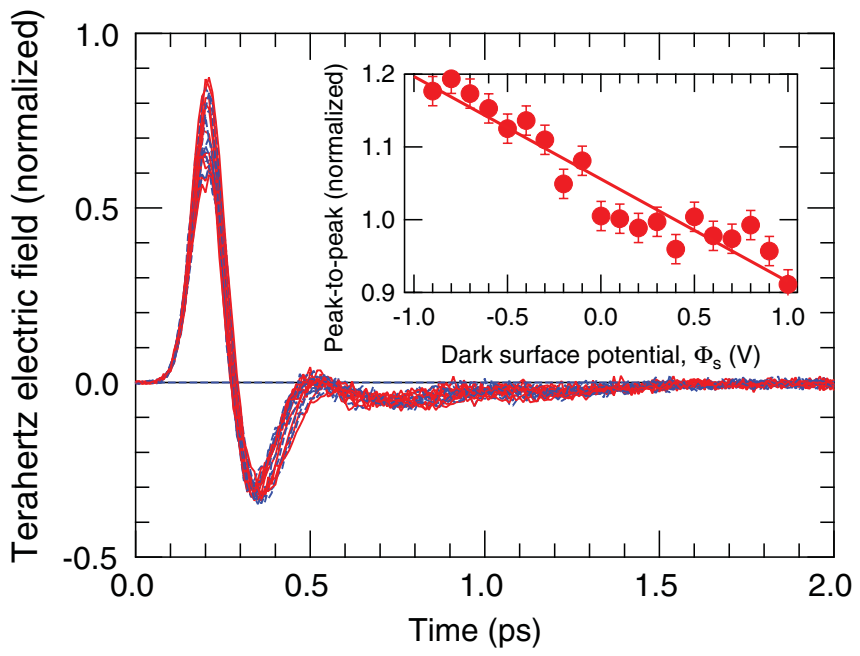

FIG. 5. (Color online) Role of surface potential in the emission of terahertz radiation from InAs. The surface potential has a systematic, but small, effect.

simple to vary the effective mass. However, our simulation allows the effect of such a modification to be quantified.

\section{G. Absorption coefficient}

The depth over which photon absorption occurs changes three features of the terahertz emission: The intensity, the peak shape, and the peak frequency (Fig. 7). The change in intensity with absorption coefficient is simple to understand: A denser distribution of electrons near the surface enhances the escape rate of reflected electrons into the bulk. The change in peak shape is a result of an altered plasma frequency of the secondary oscillation. The change in the secondary

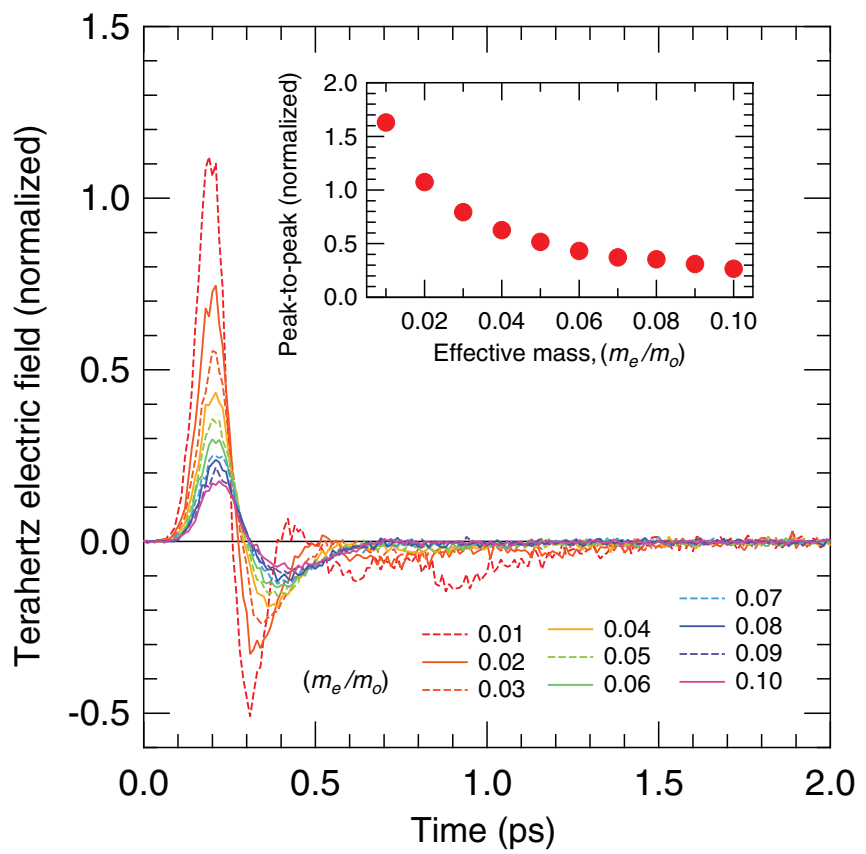

FIG. 6. (Color online) Role of electron effective mass in the $\Gamma$ valley in emission of terahertz radiation from InAs. Decreasing the effective mass strongly increases terahertz emission.

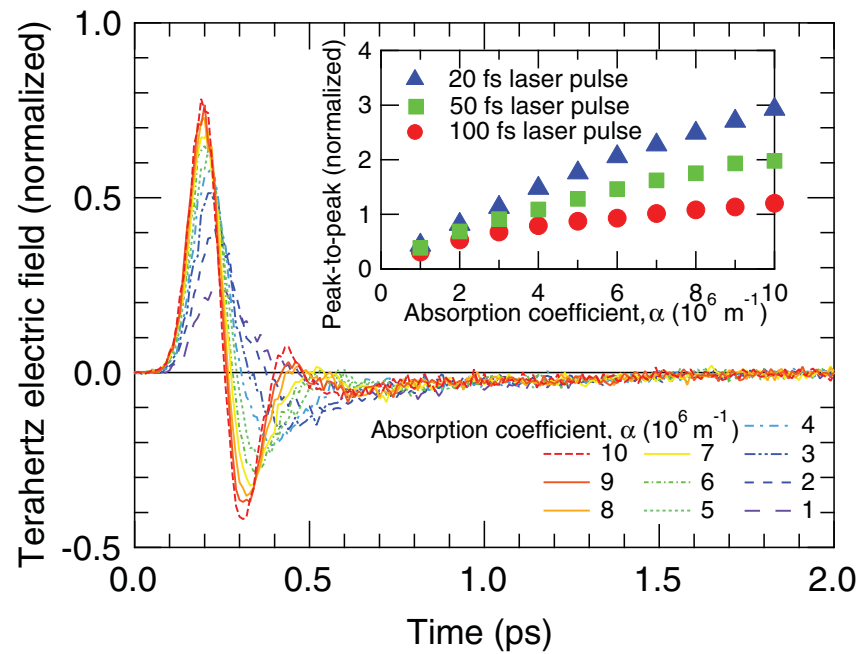

FIG. 7. (Color online) Role of absorption coefficient in the emission of terahertz radiation from InAs. Increasing the absorption coefficient strongly increases the terahertz emission.

oscillation also accounts for the shift of the frequency of the maximum radiated terahertz field. Increasing the absorption coefficient for longer laser pulses ( $\gtrsim 100 \mathrm{fs}$ ) results in the peak-peak intensity increasing to a maximum value, which then remains almost constant. This saturation effect is not observed for shorter pulses ( $\lesssim 100 \mathrm{fs})$. For shorter pulse lengths, the increase in terahertz emission remains linear with absorption coefficient to large values of absorption coefficient. We relate the unexpected saturation observed for longer pulses to the vanguard counter-potential effect described in Sec. IV.

\section{THE VANGUARD COUNTER-POTENTIAL EFFECT}

In examining the effect of various material parameters on the terahertz emission, it became clear that the terahertz emission was saturating when intuition would suggest it should continue to increase. One example was the terahertz emission saturating as the band gap decreased; another, even more marked example, was the terahertz emission saturating as the absorption coefficient increased.

We have checked that the observed saturation is not a computational artifact induced by limited temporal spatial discretization. We both doubled and halved both our time steps and voxel size. The saturation effect remains.

While reduction in emission efficiency has been noted before in relation to, for example, increasing optical fluence, ${ }^{17}$ and the screening effect of a larger number of electrons, ${ }^{43}$ the mechanism we identify here is quite different. It relates to the length of the laser pulse when this is on the scale of significant charge-carrier migration times. The effect is illustrated in Fig. 8.

The photo-Dember effect leads to the net diffusion of electrons from the surface. For long pulses $(\approx 100 \mathrm{fs})$, electrons from the leading edge of the laser pulse are able to escape sufficiently far into the InAs bulk to set up a counter-potential for electrons produced at the peak of the optical pulse. This counter-potential has the effect of reducing the overall current from the surface. Reducing the pulse length to $\approx 50 \mathrm{fs}$ alleviates this retardation. For such a short pulse, there is less time 


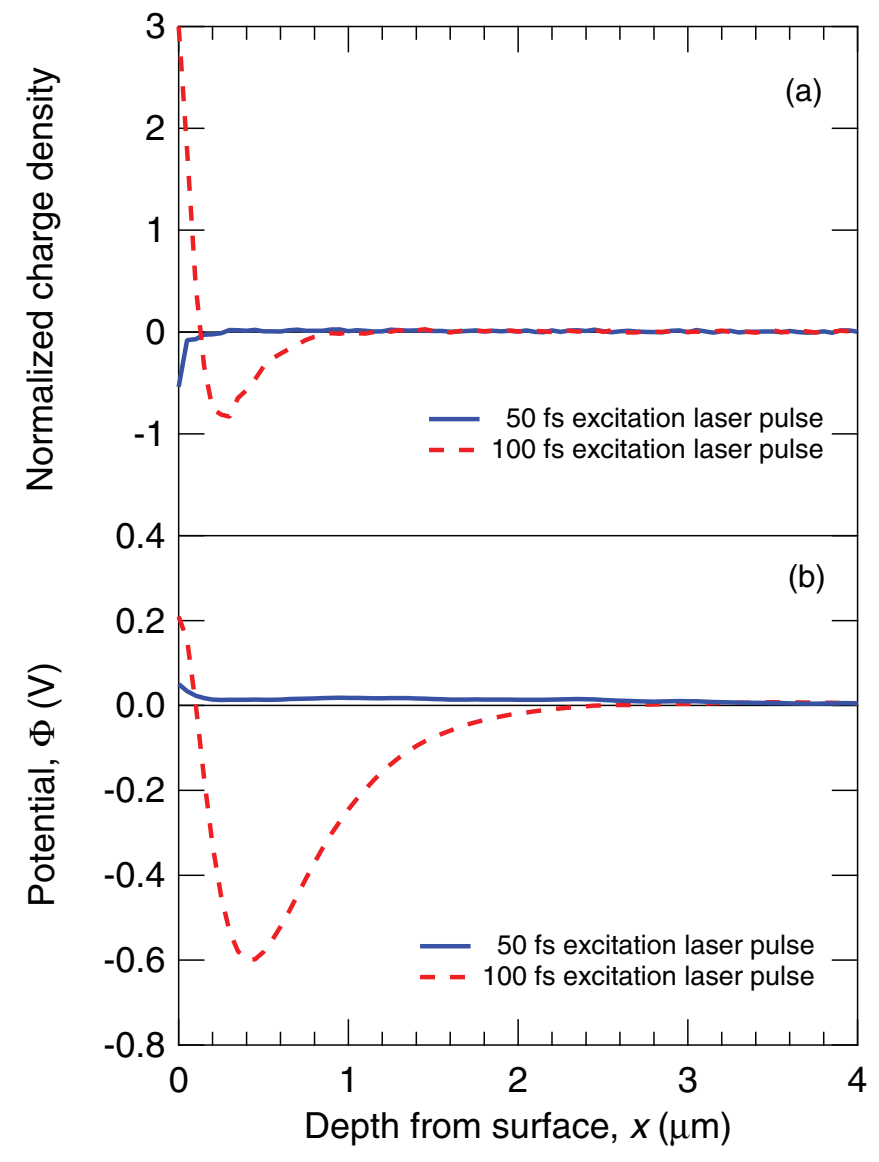

FIG. 8. (Color online) Origin of the vanguard counter-potential effect. (a) The upper panel shows the charge density for excitation laser pulses of two different durations, one of $50 \mathrm{fs}$ and the other of $100 \mathrm{fs}$, at the time when the peak of the pulse is at the surface. For the longer, $100 \mathrm{fs}$ pulse, enough electrons have already escaped from the surface to set up a charge layer within the bulk that repels the electrons being generated at the surface at the peak of the pulse. (b) The lower panel shows the electrostatic environment for the electrons at the peak injection point for two different laser pulse lengths, 50 and 100 fs. For the longer, 100 fs pulse, enough electrons have escaped from the leading edge to produce a negative potential which repels the subsequent electrons.

for electrons from the leading edge to escape sufficiently far into the surface to set up a repelling potential for subsequent electrons.

Figure 9 shows the effects of further reducing the laser pulse length to $10 \mathrm{fs}$ for two different absorption coefficients. The increase in peak-peak amplitude with decreasing laser FWHM is explained by increased current surge since

$$
E_{\mathrm{THz}} \propto \frac{d \mathbf{J}(t)}{d t} \propto \frac{\langle\mathbf{v}\rangle d N}{d t}+\frac{N d\langle\mathbf{v}\rangle}{d t},
$$

where $\langle\mathbf{v}\rangle$ is the average differential velocity of holes and electrons, and $N$ is the total number of electron-hole pairs. In our simulation all lasers pulses contain equal numbers of photons with equal energy $(1.55 \mathrm{eV})$ so the main effect of reducing the laser pulse FWHM is to increase $d N / d t$ by introducing electron-hole pairs at a faster rate. From Eq. (A6), the electron-hole pairs are created with identical kinetic energy regardless of pulse length so at first glance

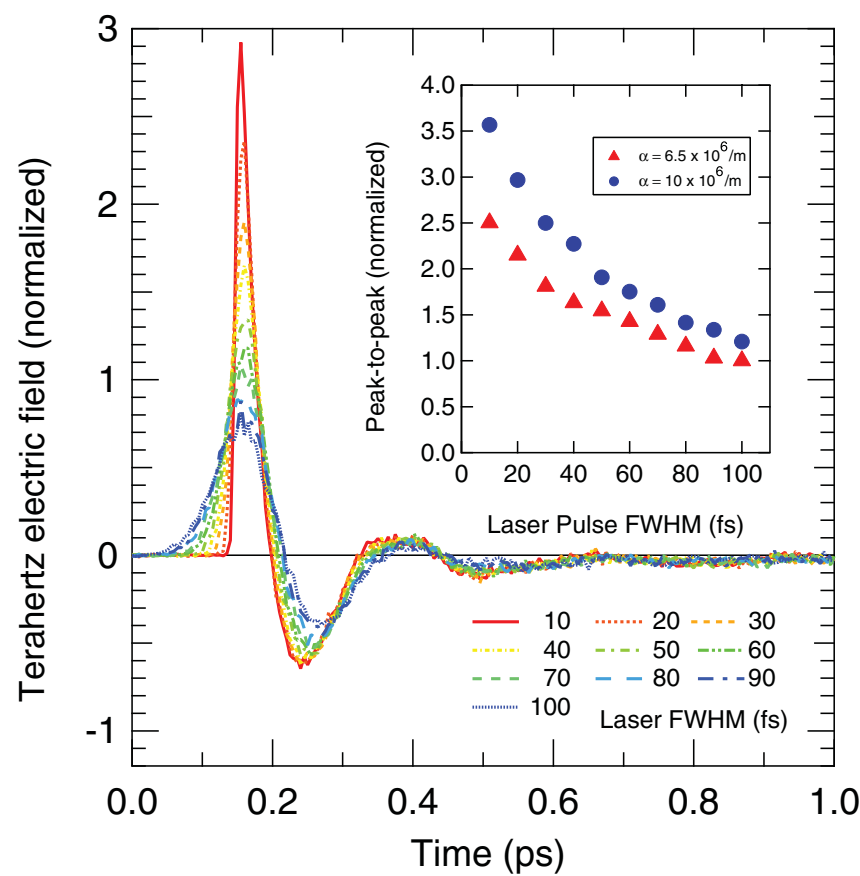

FIG. 9. (Color online) Role of laser pulse length on terahertz radiation from InAs. Inset: Comparison of the peak-to-peak intensity for different photon absorption coefficients. Reducing the absorption depth $1 / \alpha$ decreases the characteristic Dember escape time of the vanguard electrons responsible for the counter-potential effect.

$\langle\mathbf{v}\rangle$ should not depend on laser pulse length. However, the vanguard counter-potential effect provides a deacceleration mechanism as the vanguard electrons from the leading edge of the pulse form an electric dipole which naturally acts to retard subsequent photo-Dember diffusion, as in Fig. 8. The decrease in $d\langle\mathbf{v}\rangle / d t$ due to the counter-potential is enhanced for higher absorption since vanguard electrons escape from the near-surface region within a shorter time period. For this reason, for longer laser pulses (100 fs), terahertz emission is not enhanced by increased absorption. However, Fig. 9 reveals that the combination of shorter laser pulses and higher absorption led to an enhancement of the terahertz emission by 3.5 times in the simulations. In these cases electron-hole pairs were created more rapidly than the characteristic time needed to form the counter-potential. The laser pulse length relative to electron transit times through the absorption depth in the emitter material is thus a key factor in the generation of terahertz radiation. As is well known, the time-bandwidth theorem implies shorter pulses should lead to a greater bandwidth. ${ }^{7}$ Other simulations have shown previously that shorter pulses will also lead to an overall higher intensity. ${ }^{36}$ Our simulation implies that there is also a different terahertz emission dependence on material parameters, for example, effective mass, absorption coefficient, and band gap, for different pulse lengths owing to the existence of a vanguard counter-potential.

\section{CONCLUSIONS}

We have considered in detail the effects of varying distinct parameters on the emission of terahertz radiation from InAs. 
The trends we have identified would also apply to other narrowgap polar semiconductors, such as InSb. ${ }^{24,39}$ On the whole, scattering mechanisms have little effect. As expected, they reduce the radiation emitted, but the effect only amounts to a few percent. Further, in agreement with the previous literature on InAs, polar optical phonon scattering is the most important of the scattering mechanisms. Intervalley, ionized-impurity, and carrier-carrier scattering play very minor roles.

The effect of varying the dielectric constant-if that were practical-is relatively small, amounting to a maximum change in the radiated terahertz field of only about $10 \%$. A similar observation applies to varying the surface potential. Even changing the surface potential by as much as $1 \mathrm{~V}$ only results in a change in the radiated terahertz field of about $10 \%$. This confirms that the photo-Dember effect, rather than surface-field effects, is dominating the terahertz emission.

The three factors that have the most marked effects on terahertz production are the band gap, the effective mass, and the absorption coefficient. Reducing the band gap would increase the terahertz production; increasing it would result in loss of terahertz output. Decreasing the effective electron mass has a very marked effect. However, engineering either of these properties would require a new material to be designed with a drastically different band structure; this may or may not be feasible. Increasing the absorption coefficient can lead to a very dramatic increase in terahertz production, with a doubling in absorption coefficient typically leading to a $50 \%$ increase in the radiated terahertz field. It is in pursuing this, perhaps by surface engineering, where the greatest improvements in terahertz surface emission from narrow-gap semiconductors might be expected.

In pushing the limits of terahertz emission in our simulation, we observed an unanticipated saturation of emission when, for example, the absorption coefficient is increased. We attribute this to the vanguard counter-potential effect: The electrons photogenerated by the leading edge of the laser pulse have sufficient time to move far from the surface and set up a repulsive potential tending to counteract the photo-Dember diffusion by the time the main body of electrons, at the peak of the pulse, are photogenerated. This may partly explain why approaches employing the steady-state Dember effect fail to predict the reduced efficiency of InSb compared to InAs. ${ }^{24}$ While it is well known that short pulses are needed for large bandwidth, the vanguard counter-potential mechanism underscores that short pulses are crucial for strong terahertz emission.

\section{ACKNOWLEDGMENTS}

This work was supported by the Australian Research Council and by the University of Wollongong. The work was also supported by Chinese Academy of Sciences Visiting Professorship for Senior International Scientists Grant No. 2010T2J07.

\section{APPENDIX : DETAILS OF THE MODEL}

\section{Initial conditions}

The initial conditions of the system, before the laser strike $\left(t<t_{\text {laser }}\right)$, depend on the material's parameters and the temperature. Equal numbers of intrinsic holes and electrons are introduced according to

$n_{i}=p_{i}=2\left(\frac{k_{B} T}{2 \pi \hbar^{2}}\right)^{3 / 2}\left(m_{e} m_{h}\right)^{3 / 4} \exp \left(-E_{g} / 2 k_{B} T\right)$,

where $n_{i}$ is the concentration of intrinsic electrons, $p_{i}$ is the concentration of intrinsic holes, $k_{B}$ is the Boltzmann constant, $m_{e}$ is the electron effective mass, $m_{h}$ is the hole effective mass, $E_{g}$ is the energy of the band gap, and $T$ is the temperature. The concentration and sign of mobile extrinsic carriers are determined by the doping level. It is assumed that the temperature is sufficiently high $\left(k_{B} T \gg E_{i}\right.$, where $E_{i}$ is the impurity energy) that all potential extrinsic carriers have been excited from the donors or acceptors. A stationary background charge is included to model the dopant ions. The charge distribution is initially set to be uniform. The initial speed of the charge carriers is set by the Maxwell speed distribution and the directions are set randomly over $4 \pi$ steradians by selecting random polar $(\theta)$ and azimuthal $(\phi)$ angles. The simulation is then run long enough so that the initial carriers reach equilibrium with the boundary conditions and surface potential of the system. This ensures the later results are free of computational transients associated with starting the simulation at $t=0$.

\section{Calculating the electric field}

At the beginning of each time interval $d t$, the instantaneous position of each electron, hole, and immobile dopant is known. This information is used to calculate the local electric field. A finite element method is used. The simulation volume is divided into cubic cells. Each charge is assigned to the nearest cell.

Poisson's equation, relating voltage $V$ at position $\mathbf{r}$ and time $t$ to the charge density $\rho$ and relative dielectric constant $\epsilon(\infty)$,

$$
\nabla^{2} V(\mathbf{r}, t)=-\frac{\rho(\mathbf{r}, t)}{\epsilon(\infty)},
$$

is then solved by finite differencing, in effect reducing this partial differential equation into $m \times m \times n$ simultaneous equations, where $m$ is the number of cells along the edge of the square face on the surface and $n$ the number of cells running into the depth of the simulation volume. Here $m=$ 20 and $n=60$. Each cell is given a length of $0.1 \mu \mathrm{m}$, so the simulation volume is $2 \mu \mathrm{m} \times 2 \mu \mathrm{m} \times 6 \mu \mathrm{m}$.

At the extremes of the simulation volume, suitable boundary conditions must be applied. At the front surface of the volume, a Dirichlet boundary condition $\left(V=V_{\text {surface }}\right)$ is applied to allow for Fermi level pinning caused by charge trapped in surface-localized states. On the other five surfaces, a Neumann boundary condition $(\partial \Phi / \partial x=0 ; \Phi$ is the electric potential) is applied. On the four sides of the simulation volume perpendicular to the front surface, symmetrical conditions are applied, since the electric field should vanish at these surfaces by charge neutrality.

Once the boundary conditions are set, the simultaneous equations are solved by a successive over-relaxation method using Chebyshev acceleration, ${ }^{44}$ essentially a modification of the Jacobi-Strauss method. ${ }^{45}$ 
Once the electric potential is known, the electric field $\mathbf{E}$ is calculated from $\mathbf{E}=-\nabla \Phi$ by finite differencing.

\section{Ballistic motion over time interval $d t$}

Carriers inside the nondegenerate semiconductor are approximated as incoherent plane waves with a formal equation of motion given in terms of the rate of change of momentum, $\mathbf{k}$, by $\hbar \dot{\mathbf{k}}=-e \mathbf{E}$ where, for simplicity, we write this as the equivalent classical equation involving the charge $q$ and the effective mass $m^{*}$ of the charge carrier of interest:

$$
\ddot{\mathbf{r}}=\frac{q}{m^{*}}[\mathbf{E}(\mathbf{r}, t)] \text {. }
$$

The local band structure of the system primarily enters into the calculation through the effective mass, which is assumed to be constant in the nearly parabolic regions where photoexcitation (or scattering) occur.

Euler's method is then used to move electrons ballistically over the time interval $d t$. Here we use $d t=10 \mathrm{fs}$. The position and velocity are updated at each interval.

A strategy must be employed to deal with the case when a particle reaches the boundary of the simulation volume. This was done by considering the front surface to be perfectly reflecting and using cyclic boundary conditions at the other surfaces.

\section{Scattering}

To determine whether scattering occurred in a small time interval, the kinetic energy of the carrier was calculated assuming parabolic bands:

$$
E_{k}=\frac{\hbar^{2} k^{2}}{2 m^{*}} .
$$

Then the probability that the particle was scattered could be calculated using the formulations summarized in Table II.

Polar optical scattering refers to emission of polar optical phonons. Intervalley scattering allows carriers to move between the central $\Gamma$ valley and the four satellite $L$ valleys. Ionized-impurity scattering refers to scattering from fixed charges. Carrier-carrier scattering refers to scattering from mobile charges. The values for the constants used in the model are given in Table I. Figure 1 gives the scattering rates related to the different mechanisms as a function of electron energy.

Once the scattering is calculated, the new wave vector is then calculated according to the polar $(\theta)$ and azimuthal $(\phi)$ angular distributions pertinent to the given scattering mechanism.

\section{The laser strike}

At $t=t_{\text {laser }}$, the laser pulse arrives. The central photon energy, total photon energy, and pulse duration are specified as simulation parameters. A Gaussian spatial distribution was employed (but neglected if the spot size was much larger than the simulation volume and so the excitation essentially uniform across the front face). In the simulations here, the laser pulse was of $100 \mathrm{fs}$ full width at half maximum, total energy $1 \mathrm{~nJ}$, and spot size $\gg 2 \mu \mathrm{m}$.

The effect of the laser strike is to introduce electron-hole pairs. Each photon is assumed to create a single electronhole pair with a quantum efficiency of 1 and with a depth distribution given by a Beer-Lambert law:

$$
N(x)=N(0) e^{-\alpha x} .
$$

Here $N(x)$ represents the number of photons at depth $x$ and $\alpha$ is the absorption coefficient.

The energy of a photoexcited electron-hole pair at creation obeys energy conservation in spherical bands with parabolic dispersion,

$$
E_{k}=E_{\text {photon }}-E_{g}=E_{\text {hole }}+E_{\text {electron }}=\frac{m_{e} v_{e}^{2}}{2}+\frac{m_{h} v_{h}^{2}}{2} .
$$

Here the photon energy $E_{\text {photon }}=\hbar \omega_{\text {photon }}$, and $v_{e}$ and $v_{h}$ are the electron and hole speeds, respectively.

The energy is partitioned depending on relative masses so that linear momentum is conserved:

$$
v_{h}=\frac{v_{e} m_{e}}{m_{h}} .
$$

We assume that the hole and electron always are created traveling in opposite directions, chosen from an isotropic distribution, and disregard the small effects of anisotropic band structure and photon momentum.

Once introduced, the photocarriers (or hot carriers) are treated in exactly the same way as the intrinsic and extrinsic charge carriers (or cold carriers) set up in the initial conditions.

\section{Calculating the terahertz output}

The primary quantity of interest is the time behavior of the current density $\langle\mathbf{J}(t)\rangle$ subsequent to the laser strike. This is extracted as a ensemble average over all carriers including photoexcited, intrinsic, and extrinsic.

$$
\langle\mathbf{J}(t)\rangle=\sum q_{i} \mathbf{v}_{i} .
$$

The terahertz output is then proportional to $d\langle\mathbf{J}(t)\rangle / d t$.

\footnotetext{
*Currently with The Bragg Institute, Australian Nuclear Science and Technology Organisation, Lucas Heights, New South Wales 2234, Australia.

${ }^{\dagger}$ roger@uow.edu.au

${ }^{1}$ P. H. Siegel, IEEE Trans. Microwave Theory Tech. 50, 910 (2002).

${ }^{2}$ B. Ferguson and X.-C. Zhang, Nature Mater. 1, 26 (2002).
}

\footnotetext{
${ }^{3}$ R. A. Lewis, Proc. IEEE 95, 1641 (2007).

${ }^{4}$ R. A. Lewis, Am. J. Phys. 79, 341 (2011).

${ }^{5} \mathrm{D}$. Mittleman, editor, Sensing with Terahertz Radiation (Springer, Berlin, 2003).

${ }^{6}$ K. Sakai, editor, Terahertz Optoelectronics (Springer, Berlin, 2005).

${ }^{7}$ S. L. Dexheimer, editor, Terahertz Spectroscopy: Principles and Applications (CRC Press, Boca Raton, 2008).
} 
${ }^{8}$ S. D. Ganichev and W. Prettl, Intense Terahertz Excitation of Semiconductors (Oxford University Press, Oxford, 2006).

${ }^{9}$ Y.-S. Lee, Principles of Terahertz Science and Technology (Springer, Berlin, 2009).

${ }^{10} \mathrm{X}$.-C. Zhang and J. Xu, Introduction to $\mathrm{THz}$ Wave Photonics (Springer, New York, 2010).

${ }^{11}$ X. C. Zhang, B. B. Hu, J. T. Darrow, and D. H. Auston, Appl. Phys. Lett. 56, 1011 (1989).

${ }^{12}$ C. A. Schmuttenmaer, Chem. Rev. 104, 1759 (2004).

${ }^{13}$ V. L. Malevich, R. Adomavičius, and A. Krotkus, C. R. Phys. 9, 130 (2008).

${ }^{14}$ P. Smith, D. Auston, and M. Nuss, IEEE J. Quantum Electron. 24, 255 (1988).

${ }^{15}$ C. Fattinger and D. Grischkowsky, Appl. Phys. Lett. 54, 490 (1989).

${ }^{16}$ M. R. Stone, M. Naftaly, R. E. Miles, J. R. Fletcher, and D. P. Steenson, IEEE Trans. Microwave Theory Tech. 52, 2420 (2004).

${ }^{17}$ T. Hattori, K. Tukamoto, and H. Nakatsuka, Jpn. J. Appl. Phys. 40, 4907 (2001).

${ }^{18}$ V. L. Malevich, A. Krotkus, A. Bičiūnas, and V. Pačebutas, J. Appl. Phys. 104, 113117 (2008).

${ }^{19}$ M. Reid, I. V. Cravetchi, and R. Fedosejevs, Phys. Rev. B 72, 035201 (2005).

${ }^{20}$ S. Hargreaves and R. A. Lewis, Appl. Phys. Lett. 93, 242101 (2008).

${ }^{21}$ J. Lloyd-Hughes, E. Castro-Camus, M. D. Fraser, C. Jagadish, and M. B. Johnston, Phys. Rev. B 70, 235330 (2004).

${ }^{22}$ R. Mendis, M. L. Smith, L. J. Bignell, R. E. M. Vickers, and R. A. Lewis, J. Appl. Phys. 98, 126104 (2005).

${ }^{23}$ M. B. Johnston, D. M. Whittaker, A. Corchia, A. G. Davies, and E. H. Linfield, Phys. Rev. B 65, 165301 (2002).

${ }^{24}$ P. Gu, M. Tani, S. Kono, K. Sakai, and X.-C. Zhang, J. Appl. Phys. 91, 5533 (2002).

${ }^{25}$ V. L. Malevich, Acta Phys. Pol. A 107, 169 (2005).
${ }^{26}$ M. Nakajima, Y. Oda, and T. Suemoto, Appl. Phys. Lett. 85, 2694 (2004).

${ }^{27}$ D. Liu and Y. Tan, Int. J. Infrared Millimeter Waves 26, 1265 (2005).

${ }^{28}$ V. L. Malevich, Surf. Sci. 454, 1074 (2000).

${ }^{29}$ V. L. Malevich, Semicond. Sci. Technol. 17, 551 (2002).

${ }^{30}$ M. B. Johnston, D. M. Whittaker, A. Corchia, A. G. Davies, and E. H. Linfield, J. Appl. Phys. 91, 2104 (2002).

${ }^{31}$ D. Liu and J. Qin, Int. J. Infrared Millimeter Waves 24, 2127 (2003).

${ }^{32}$ W. Fawcett, A. D. Boardman, and S. Swain, J. Phys. Chem. Solids 31, 1963 (1970).

${ }^{33}$ C. Jacoboni and L. Reggianni, Rev. Mod. Phys. 55, 645 (1983).

${ }^{34} \mathrm{C}$. Jacoboni and P. Lugli, The Monte Carlo Method for Semiconductor Device Simulation (Springer-Verlag, New York, 1989).

${ }^{35}$ K. Brennan and K. Hess, Solid-State Electron. 27, 347 (1984).

${ }^{36}$ E. Castro-Camus, J. Lloyd-Hughes, and M. B. Johnston, Phys. Rev. B 71, 195301 (2005).

${ }^{37} \mathrm{~S}$. Adachi, Properties of Group-IV, III-V and II-VI Semiconductors (Wiley, West Sussex, England, 2005).

${ }^{38} \mathrm{P}$. Yu and M. Cardona, Fundamentals of Semiconductors: Physics and Material Properties (Springer, New York, 2001).

${ }^{39}$ G. Molis, R. Adomavičius, and A. Krotkus, Physica B 403, 3786 (2008).

${ }^{40}$ X. L. Wang, Phys. Rev. Lett. 100, 156404 (2008).

${ }^{41}$ K. Radhanpura, S. Hargreaves, R. A. Lewis, and M. Henini, Appl. Phys. Lett. 94, 251115 (2009).

${ }^{42}$ Z. W. Deng, R. W. M. Kwok, W. M. Lau, and L. L. Cao, Appl. Surf. Sci. 158, 58 (2000).

${ }^{43}$ K. Liu, J. Xu, T. Yuan, and X.-C. Zhang, Phys. Rev. B 73, 155330 (2006).

${ }^{44} \mathrm{~W}$. H. Press, Numerical Recipes in C: The Art of Scientific Computing (Cambridge University Press, Cambridge, 1989).

${ }^{45}$ J. Thijssen, Computational Physics (Cambridge University Press, Cambridge, 2007). 